\title{
Correlation of Ki-67 and MCM-2 proliferative marker expression with grade of histological malignancy (G) in ductal breast cancers
}

\author{
Andrzej Wojnar ${ }^{1}$, Christopher Kobierzycki ${ }^{2}$, Anna Krolicka ${ }^{2}$, Bartosz Pula ${ }^{2}$, \\ Marzena Podhorska-Okolow ${ }^{2}$, Piotr Dziegiel ${ }^{1,2,3}$
}

\author{
${ }^{1}$ Lower Silesian Oncology Centre, Wroclaw, Poland \\ ${ }^{2}$ Department of Histology and Embryology, Medical University, Wroclaw, Poland \\ ${ }^{3}$ Department of Histology and Embryology, Medical University, Poznan, Poland
}

\begin{abstract}
The study aimed at examining a relationship between expression of Ki-67 antigen and minichromosome maintenance 2 protein (MCM-2) and a grade of histological malignancy $\mathrm{G}$ in ductal breast cancers. The function of widely used marker of proliferation Ki-67 is still not clear. In contrast, the MCM-2 protein is well known to play an important role in controlling the cell cycle. Both proteins represent small protein molecules, which manifest nuclear expression only during cell division of normal and neoplastic cells. Their expression is noted in several malignant tumours. These studies were conducted on 56 archival paraffin blocks of ductal breast cancers. Immunohistochemical reactions were performed using monoclonal Ki-67- and MCM-2-specific antibodies. Statistical analysis demonstrated a positive correlation between expressions of two proteins $(\mathrm{r}=0.6 ; \mathrm{p}<0.05)$. The most intense expression of these two markers was demonstrated in G3 grade cancers. Statistical analysis showed more pronounced expression of Ki-67 antigen in G3 grade cancers as compared to cancers of G1 and $\mathrm{G} 2$ grades $(\mathrm{p}<0.001)$ and, in the case of MCM-2 protein, a more pronounced expression in G3 grade cancers, as compared to those of G1 $(\mathrm{p}<0.05)$ or $\mathrm{G} 2$ grade $(\mathrm{p}<0.01)$. The results obtained in our study suggest that MCM- 2 could be used as a marker of proliferation in breast carcinomas.
\end{abstract}

Key words: Breast cancer, Ki-67, MCM-2.

\section{Introduction}

In 2006, 429900 cases of breast cancer were diagnosed in Europe (the most frequent malignant tumour, $13.5 \%$ of all cancers), and the respective mortality reached 131900 cases [1]. In 2008, in turn, 182460 cases of breast cancer were detected in the United States, with the mortality of 40480 cases. In parallel with pulmonary cancer and colorectal carcinoma, this was one of the three most frequent malignant tumours, accounting together for, around, $50 \%$ of neoplastic diseases in USA, representing the most frequent cause of death of women 20 to 59 years of age [2]. In view of the above, an early diagnosis and effective treatment of disease are immensely important. Intensive studies are

Correspondence: Piotr Dziegiel, Dept. of Histology and

Embryology, Medical University, Chalubinskiego 6a, 50-368 Wroclaw, Poland; tel.: (+4871) 7841354,

fax.: (+4871) 7840082, e-mail: piotr@hist.am.wroc.pl being carried out on determining reliable prognostic factors of a high specificity and sensitivity which could increase efficiency of treatment in such cases. Markers of a significant prognostic value manifest a strict correlation between intensity of their expression and prognosis. In clinical practice cases frequently occur, which manifest similar pathological characters but differ from each other in the course of the disease and in sensitivity to the applied treatment procedures. While evaluation of Ki-67 antigen expression is common in prognostic analysis of various tumour types (including soft tissue tumours, breast cancers, lung or prostate, brain tumours), examination of MCM-2 protein expression in such tumours, including breast cancer, still remains in its preliminary phase $[4,7]$.

$\mathrm{Ki}-67$ belongs to the group of most frequently estimated proliferation markers but its value as a prognostic marker has not been sufficiently proven and studies which dealt with the relationship between cell proliferation and clinical course of a disease fre- 
Table 1. Clinical and pathological characteristic of studied patients.

\begin{tabular}{|c|r|}
\hline Clinical/pathological parameter & \multicolumn{1}{|c|}{$\mathrm{N}(\%)$} \\
\hline Mean age in years (range) & $54.93(35-83)$ \\
\hline Tumour size & \\
T1 & $35(62.5 \%)$ \\
T2 & $18(32.1 \%)$ \\
T3 & $3(5.4 \%)$ \\
\hline Lymph nodes & \\
pN0 & $34(60.7 \%)$ \\
pN1 & $9(16.1 \%)$ \\
pN2 & $8(14.3 \%)$ \\
pN3 & $5(8.9 \%)$ \\
\hline Clinical advencement & \\
I & $34(60.7 \%)$ \\
II & $9(16.1 \%)$ \\
IV & $13(23.2 \%)$ \\
Histol ogical differentiation (Grade) & 0 \\
G1 & $7(12.5 \%)$ \\
G2 & $28(50 \%)$ \\
G3 & $21(37.5 \%)$ \\
\hline
\end{tabular}

quently yielded divergent results [3-8]. During interphase Ki-67 co-localizes with perinuclear located DNA and DNA located near the nuclear envelope what suggests that it is involved in perinuclear chromatin organization [9]. Expression of Ki-67 may develop also in situations of an inhibited DNA synthesis or when the cell undergoes the process of apoptosis [10]. Since both biological functions of $\mathrm{Ki}-67$ and its significance as a prognostic factor remain unclear, it has been decided to include into the prognostic routine another proliferation marker, the MCM-2 protein. Until today, its estimation has been restricted exclusively to investigative purposes and it has not been applied in clinical and pathological evaluations. Its indubitable advantage includes the fact that biology of the factor is well recognized. MCM-2 belongs to the family of MCM (minichromosome maintenance) proteins, which includes the homogenous group of six proteins (MCM-2 to 7), responsible for the start and maintenance of replication [11-12]. At the early G1 phase of the cell cycle, MCM proteins are responsible for formation of the pre-replication complex, what differs them from the Ki-67 antigen, which is mostly present in late G1, S, G2 stages of the cell cycle $[14,15]$. The contained in the complex MCM proteins manifest activity of helicase, which disentangles DNA threads during replication process [15]. In the course of replication, this provides an appropriate access to all regions of DNA. At the $\mathrm{S}$ phase of the cell cycle MCM proteins irreversibly bind to the chromatin, which warrants that exactly one genetic materi- al replication event occurs per a single cell division [16-17]. In view of the above, expression of MCM family proteins, including MCM-2 proteins, may in future be contemplated to be used as a new proliferation marker since its expression is elevated in rapidly dividing cells [18-20]. This study attempted to examine correlation between expressions of MCM-2 protein and Ki-67 proliferation antigen and to compare their expression in ductal breast cancers of different histological malignancy grades $(G)$.

\section{Material and methods}

Patients. The studies were performed on 56 archival paraffin blocks containing fragments of invasive ductal breast cancers, sampled in 2002 during procedures of mammectomy in the Lower Silesia Oncology Centre in Wroclaw. Age of the patients ranged from 35 to 83 years (the mean of $54.93 \pm 10.87$ SD years), all the patients were of female sex. The histological malignancy grade $(\mathrm{G})$ was determined using guidelines suggested by WHO [21]. The clinical and pathological data were obtained from archives of the Lower Silesia Oncology Centre in Wroclaw (Table 1).

Immunohistochemistry. Tissue samples were fixed in $10 \%$ buffered formalin, dehydratated and embedded in paraffin. In each case, hematoxylin and eosin-stained preparations were subjected to histopathological evaluation by two pathologists. Formalin-fixed, paraffin-embedded tissue was freshly cut $(4 \mu \mathrm{m})$. The paraffin sections were mounted on Superfrost slides (Menzel Gläser, Germany), dewaxed with xylene and gradually re-hydrated. Activity of endogenous peroxidase was blocked by $30 \mathrm{~min}$ exposure to $1 \%$ $\mathrm{H}_{2} \mathrm{O}_{2}$. The IHC reactions were performed using monoclonal antibodies against MCM-2, originating from Novocastra (clone CRCT2.1, 1:50, Novocastra Laboratories Ltd, Balliol Bussiness Park West, Benton Lane, Newcastle upon Tyne, NE12 8EW, UK) and against Ki-67 antibody, originating from Dako (clone MIB-1, 1:50, Dako, Denmark A/S, Produktionsvej 42, DK-2600 Glostrup, Denmark). The antibody was diluted in antibody diluent with the background reducing component. All the reactions were accompanied by the respective control reactions in which specific antibodies were substituted by the Universal Negative Control for N-series Mouse Primary Antibodies Kit, N1698 (Dako). The studied paraffin sections were boiled in the Antigen Retrieval Solution in microwave oven in order to unblock antigenic determinants. The investigated antigens were visualized using biotinylated antibodies, streptavidin-biotinylated peroxidase complex (LSAB, Dako) and diaminobenzidine (DAB, Dako). All the sections were counterstained with Meyer's hematoxylin (20 second).

Evaluation of the intensity of IHC reaction. The intensity of Ki67 antigen and MCM-2 protein expression was evaluated using computer-assisted image analysis. In every section three fields were selected with the highest number of tumour cells yielding positive reaction /Hot Spots/. Percentage of positive cells in Hot Spots was evaluated scoring the brown-labelled cells nuclei, detected after screening of all cell nuclei under $\times 400$ magnification (Olympus $\mathrm{BX}-42$ light microscope with the visual mode AnalySis 3.2 software for computer-assisted image analysis). The general result for every sample was an average of the three hot spot percentages. The intensity of the IHC reactions was independently evaluated in coded preparations by two pathologists (AW, CK); in doubtful cases a re-evaluation was performed using a double-headed microscope and the staining was discussed until a consensus was achieved. The examining pathologists knew no clinical details related to the respective patient. 

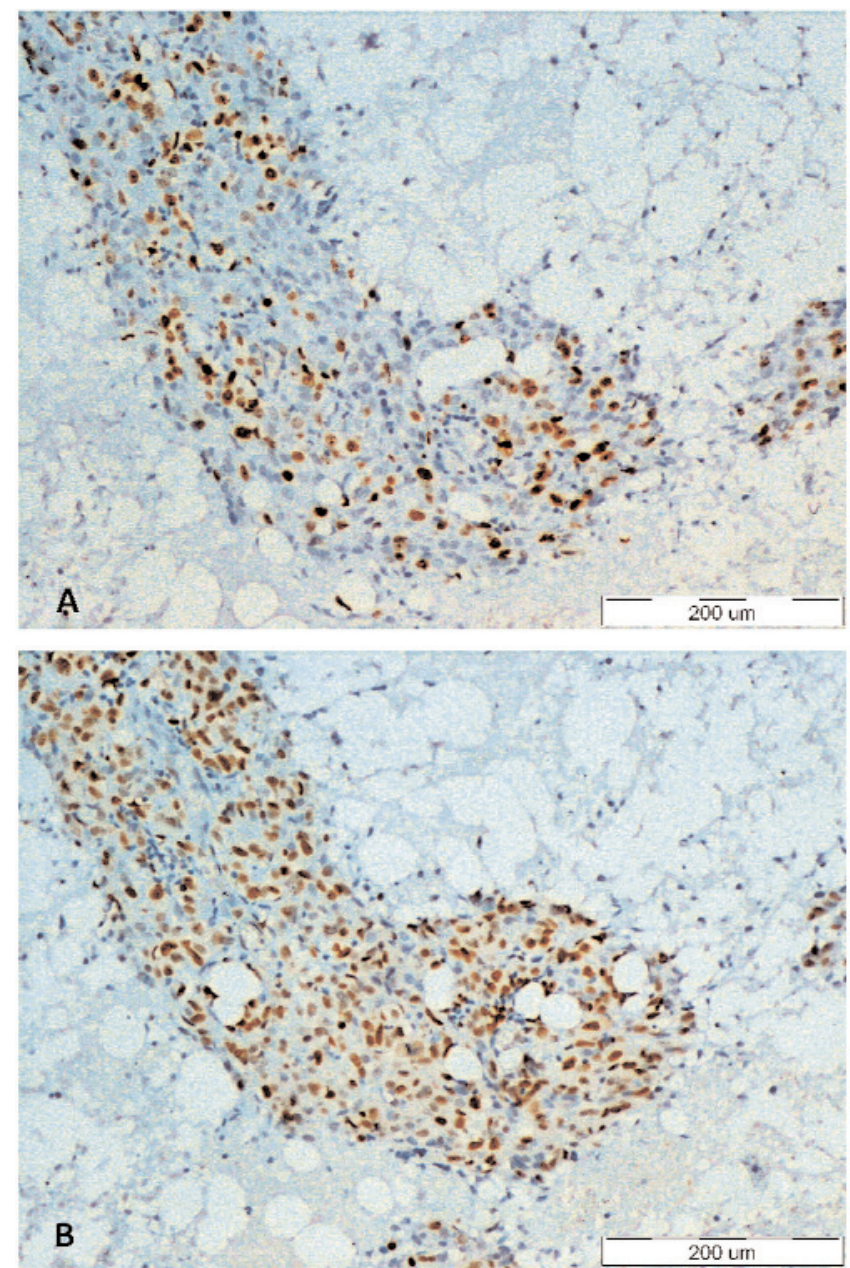

Fig. 1. Nuclear expression of Ki-67 (A) and MCM-2 protein (B) in ductal breast cancer. Serial sections, counterstaining with haematoxylin (original magnification $\times 100$ ).

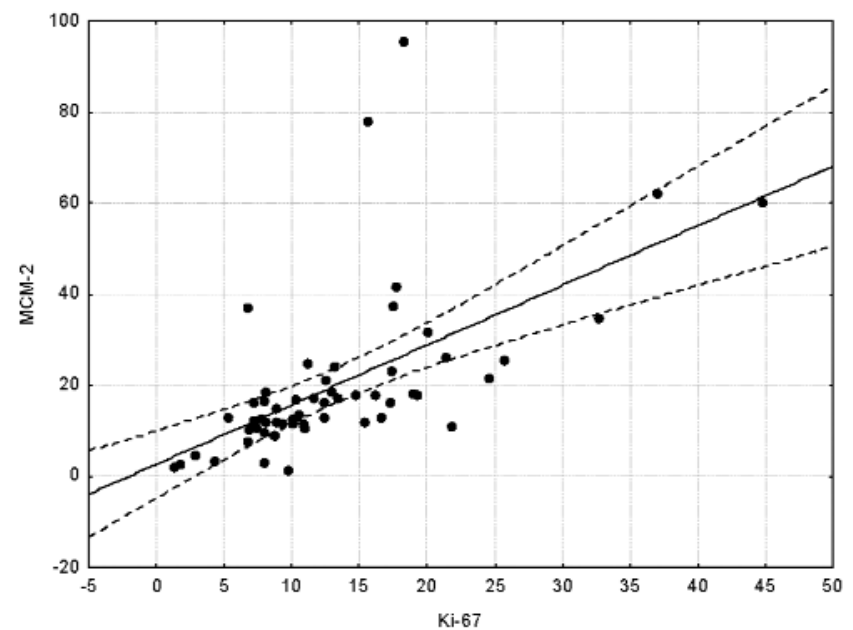

Fig. 2. Correlation of Ki-67 antigen expression with MCM-2 protein expression in ductal breast cancer: $r=0.6 ; p<0.05$.

Statistical analysis. The results were subjected to statistical analysis using Statistica 7.1 PL software (Statsoft, Poland). The relationship between the expression of $\mathrm{Ki}-67$ antigen and MCM-2 protein was
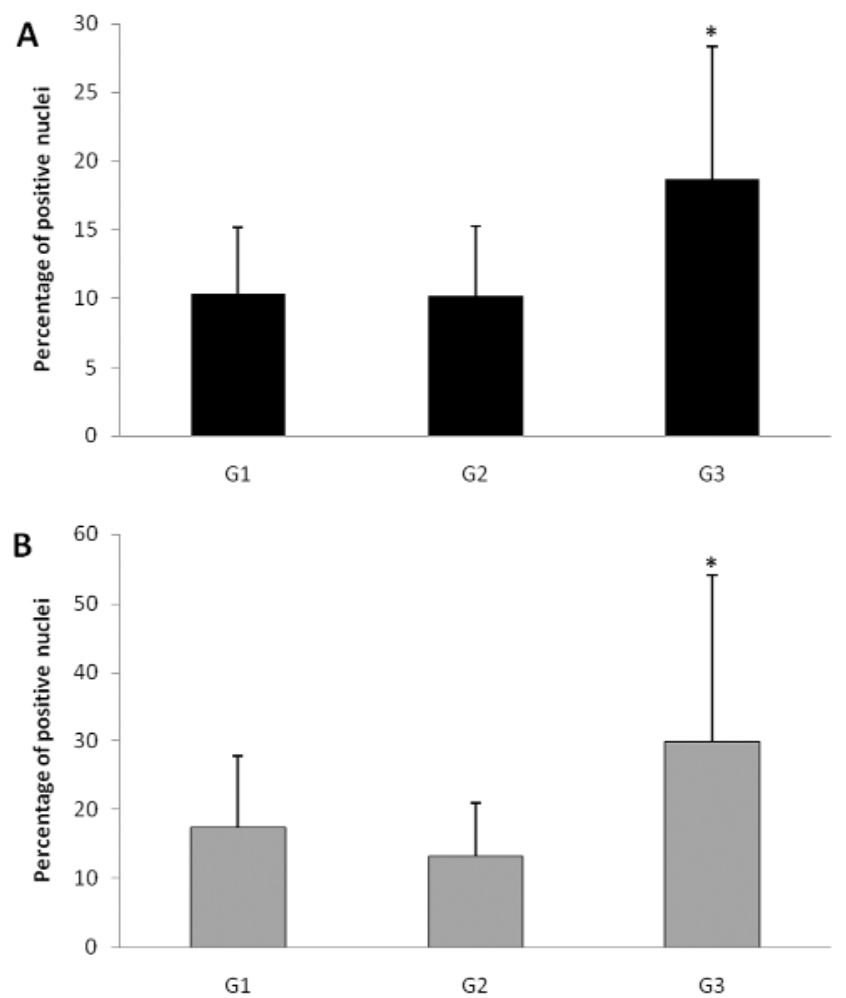

Fig. 3. Intensity of Ki-67 antigen expression ${ }^{*} \mathrm{p}<0.001 \mathrm{G} 3$ vs. G1 and G2 (A) and of MCM-2 protein: ${ }^{*} \mathrm{p}<0.05$ G3 vs. G1 and G2 (B) in ductal breast cancers.

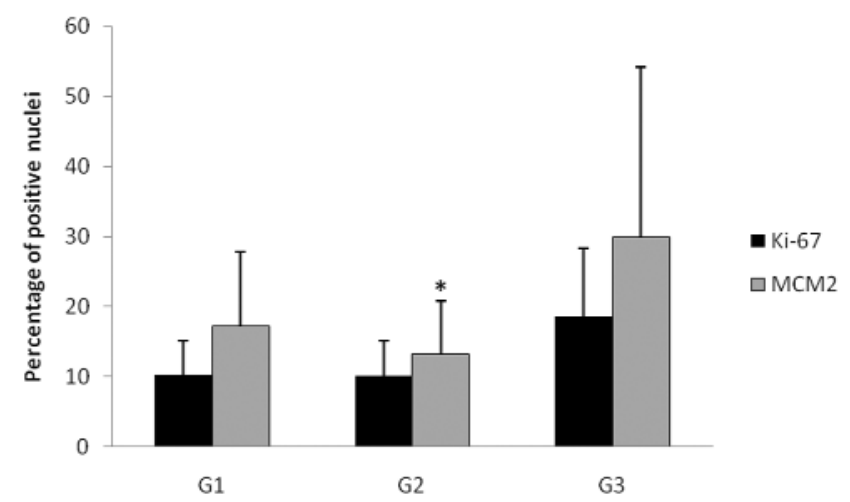

Fig. 4. Comparison between expression of Ki-67 antigen and that of MCM-2 protein in ductal breast cancers manifesting various grades of histological malignancy $\mathrm{G}$; ${ }^{*} \mathrm{p}<0.05 \mathrm{G} 2$ : Ki-67 vs. MCM2.

examined using Spearman's rank correlation. The relationship between expression intensity of the two proteins and histological malignancy grade $\mathrm{G}$ was examined using ANOVA rank test of Kruskal-Wallis. The differences were accepted as significant at $\mathrm{p}<0.05$.

\section{Results}

All the examined tumours manifested nuclear expression of Ki-67 antigen (Fig. 1A) and MCM-2 protein (Fig. 1B). In hematoxylin-counterstained serial sec- 
tions intensely brown-stained cell nuclei were noted. Statistical analysis of the relationship between expression of the two markers Ki-67 and MCM-2 demonstrated a strong positive correlation between expressions of the two markers $(r=0.6 ; \mathrm{p}<0.05)$ (Fig. 2). Also the relationship was examined between expression of the examined proteins and $\mathrm{G}$ grade of studied tumours. A more pronounced expression of Ki-67 antigen was demonstrated in G3 than that in G1 or G2 $(\mathrm{p}<0.001)$ (Fig. 3A) and, in the case of MCM-2 protein in G3 than that in $\mathrm{G} 1(\mathrm{p}<0.05)$ or $\mathrm{G} 2(\mathrm{p}<0.01)$ (Fig. 3B). For either marker the difference between expression in G1 and $\mathrm{G} 2$ proved to be insignificant. Also, expressions of Ki-67 antigen and MCM-2 protein were compared between individual grades of histological malignancy (G) of the studied tumours. Only in tumours of G2 grade expression of Ki-67 antigen was significantly lower than expression of MCM-2 protein (Fig. 4). In the case of Ki-67 antigen the average of $11 \%$ tumour cells manifested its expression in G1, 10\% in G2 and $18 \%$ in G3 while in the case of MCM-2 protein the mean percentage of positive tumour cells amounted to $17 \%$ in $\mathrm{G} 1,13 \%$ in $\mathrm{G} 2$ and $30 \%$ in $\mathrm{G} 3$.

\section{Discussion}

Expression of Ki-67 and MCM-2 markers and relationships between the two were examined in relatively numerous studies. Expression of the proteins was studied in cancers of the larynx, oral cavity, thyroid gland, in tumours of salivary gland, colorectal cancers, tumours of adrenal glands, lungs and in renal carcinoma [22-31,33]. Results of some investigators suggested a higher prognostic value of MCM-2 protein than that manifested by currently widely used Ki-67 $[23,28,30,34,35]$.

Chatrath et al. [22] examined benign and malignant lesions in the larynx. Their statistical analysis demonstrated a very strong correlation between expression of Ki-67 and that of MCM-2, which pointed to a similar prognostic value of the studied proteins. Szelachowska et al. [23] studied spinocellular cancers of oral cavity and demonstrated a higher prognostic value of MCM2 as compared to that of Ki-67. In the cases of thyroid tumours, Mehrorta et al. [24] observed increased expression of both markers in malignant lesions (follicular and papillary tumours). The studies demonstrated also that neither MCM-2 nor Ki-67 could be applied for distinguishing benign from malignant lesions in the thyroid gland. Giaginis et al. (25) showed that expression of MCM-2 was significantly linked to clinical and pathological data and manifested strong correlation with cell proliferation in colon carcinoma. In turn, studies of Guzińska-Ustymowicz et al. [26] on colorectal cancers demonstrated that an increase in MCM-2 expression might point to devel- opment of metastases to lymph nodes. In other studies Vargas et al. [27] showed in tumours of salivary glands that MCM-2 was a strong marker of differentiation between benign and malignant lesions. Szajerka et al.[28] compared expression of MT, Ki-67 and MCM-2 in tumours of adrenal cortex. They demonstrated a very strong correlation between MCM-2 and MT and a moderate correlation between MCM-2 and $\mathrm{Ki}-67$. In view of their results MCM-2 seems to provide a reliable indicator of malignant processes in adrenal cortex. In cases of pulmonary adenocarcinomas Hashimoto et al. [29] demonstrated a similar diagnostic usefulness of both Ki67 and MCM-2. On the other hand, Yang et al. [30] suggested that in non-small cell lung carcinomas two markers in parallel, MCM-2 and gesoline should be estimated. In such tumour the authors found that usage of Ki67 manifested no prognostic value. In renal carcinoma, in turn, Dudderidge et al. [31] suggested that MCM-2 should be used as a marker of proliferation and prognosis. Moreover, Kodani et al. [32] suggested that an increased expression of MCM-2 and $\mathrm{p} 53$ may point to a malignant neoplastic transformation in cells of oral mucosa. They tried to explain it that the MCM-2 expression could involve the $\mathrm{p} 53$ protein-dependent pathway and, in parallel, they found that MCM-2 represents a useful marker of proliferation.

Expression of MCM-2 protein was already studied in some papers concerning breast cancer. Reena et al. [34] examined MCM-2 expression in benign and malignant lesions of the breast. Similarly to us, they noted a high MCM-2 proliferation index in tumours manifesting grade $\mathrm{G} 3$ of histological malignancy and a relatively low index in tumours of G1 grade, pointing to positive correlation between MCM-2 expression and $\mathrm{G}$ grade. Gonzales et al.[35] obtained very similar results in studies on breast cancers, indicating that expression of MCM-2 was a more frequent and more pronounced than those of the widely used Ki-67. They postulated that MCM-2 labelling index represents a strong independent prognostic factor and for a more reliable prognostic evaluation it should be considered in parallel with parameters of the Nottingham Prognostic Index (NPI). Ki-67 and MCM-2 were also evaluated in advanced gastric cancer. Although this study did not show any correlation between both markers, shorter postoperative survival time was associated with high MCM-2 expression [33].

Results of our studies have demonstrated an increased nuclear expression of both MCM-2 protein and Ki-67 antigen in ductal breast cancers. Results obtained by us and other authors comparing expression of the two proteins point to a comparable value of the two markers, sometimes suggesting a higher significance of MCM-2. Ki-67 antigens represents the widely used proliferation marker in mammary gland cancers but there is lack of explanation related to the mecha- 
nisms in which the protein acts suggest a search for other prognostic factors. The difference in expression of both markers between G1 and G2 grades was insignificant. In view of the strong correlation in expression of the studied markers the potential of a more frequent determination of MCM-2 antigen could be considered, as a marker of proliferation in breast malignant tumours. This may be particularly valuable in ductal breast cancers since expression of the protein correlates with its $\mathrm{G}$ malignancy grade.

\section{References}

[1] Ferlay J, Autier P, Boniol M. et al. Estimates of the cancer incidence and mortality in Europe in 2006. Ann Oncol. 2007;18:581-592.

[ 2] Jemal A, Siegel R, Ward E. et al. Cancer Statistics, 2008. CA Cancer J Clin. 2008;58:71-96.

[ 3] Dziegiel P, Forgacz J, Suder E. et al. Prognostic significance of metallothionein expression in correlation with Ki-67 expression in adenocarcinomas of large intestine. Histol Histopathol. 2003;18:401-407.

[4] Dziegiel P, Salwa-Zurawska W, Zurawski J. et al. Prognostic significance of augmented metallothionein expression correlated with Ki-67 antigen expression in selected soft tissue sarcomas. Histol Histopathol. 2005;20:83-89.

[5] Krecicki T, Jelen M, Zalesska-Krecicka M. et al. Epidermal growth factor receptor (EGFR), proliferating cell nuclear antigen (PCNA) and Ki-67 antigen in laryngeal epithelial lesions. Oral Oncol. 1999;35:180-186.

[ 6] Ofner D, Grothaus A, Riedmann B. MIB-1 in colorectal carcinomas: its evaluation by three different methods reveals lack of prognostic significance. Anal Cell Path. 1999;12:61-70.

[ 7] Tungekar MF, Gatter KC, Dunnill MS. et al. Ki-67 immunostaining and survival in operable lung cancer. Histopathology. 1991;19:545-550.

[ 8] Bettendorf O, Herremann G. Prognostic relevance of Ki-67 antigen expression in 329 cases of oral squamous cell carcinoma. ORL J Otorhinolaryngol Relat Spec. 2002;64:200-205.

[9] Cheutin T, O'Donohue MF, Beorchia A. et al. Three-dimensional organization of pKi-67: a comparative fluorescence and electron tomography study using FluoroNanogold. $J$ Histochem Cytochem. 2003;51:1411-1423.

[10] Tye BK. Mcm proteins in DNA replication. Annu Rev Biochem. 1999;68: 649-686.

[11] Kearsey SE, Labib K. MCM proteins: evolution, properties and role in DNA replication. Biochim Biophys Acta. 1998;1398:113-136.

[12] Forsburg SL. Eukaryotic MCM proteins: beyond replication initiation. Microbiol Mol Biol Rev. 2004;68:109-31.

[13] Ritzi M, Knippers R. Initiation of genome replication: assembly and disassembly of replication-competent chromatin. Gene.2000;245:13-20.

[14] Laskey RA, Madine MA. A rotary pumping model for helicase function of MCM proteins at a distance from replication forks. EMBO Rep 2003;4: 26-30.

[15] Todorov IT, Attaran A, Kearsey SE. BM28, a human member of the MCM2-3-5 family, is displaced from chromatin during DNA replication. J Cell Biol. 1995;129:1433-1445.

[16] Romanowski P, Madine MA. Mechanisms restricting DNA replication to once per cell cycle: the role of Cdc 6 and ORC. Trends Cell Biol. 1997;7: 9-10.

[17] Stoeber K, Mills AD, Kubota Y. et al. Cdc6 protein causes premature entry into S-phase in a mammalian cell-free system. EMBO J. 1998;17:7219-7229.
[18] Williams RS, Shohet RV, Stilman B. A human protein related to yeast Cdc6p. Proc Natl Acad Sci USA. 1997;94:142-147.

[19] Yan Z, DeGregori J, Shohet R. et al. Cdc6 is regulated by E2F and is essential for DNA replication in mammalian cells. Proc Natl Acad Sci USA. 1998;95:3603-3608.

[20] Stoeber K, Tlsty TD, Happerfield L. et al. DNA replication licensing and human cell proliferation. $J$ Cell Sci. 2001;114:2027-2041.

[21] Tavassoli F.A., Devilee P. (Eds.). World Health Organization Classification of Tumours. Pathology and Genetics of Tumours of the Breast and Female Genital Organs. IARC Press: Lyon 2003.

[22] Chatrath P, Scott IS, Morris LS. et al. Aberrant expression of minichromosome maintenance protein-2 and Ki67 in laryngeal squamous epithelial lesions. Br J Cancer. 2003;89:10481054.

[23] Szelachowska J, Dziegiel P, Jelen-Krzeszewska J. et al. Mcm2 protein expression predicts prognosis better than Ki-67 antigen in oral cavity squamocellular carcinoma. Anticancer Res. 2006;26:2473-2478.

[24] Mehrotra P, Gonzalez MA, Johnson SJ. et al. Mcm-2 and Ki67 have limited potential in preoperative diagnosis of thyroid malignancy. Laryngoscope. 2006;116:1434-1438.

[25] Giaginis C, Georgiadou M, Dimakopoulou K. et al. Clinical significance of MCM-2 and MCM-5 expression in colon cancer: association with clinicopathological parameters and tumor proliferative capacity. Dig Dis Sci. 2009;54:282-91.

[26] Guzińska-Ustymowicz K, Pryczynicz A, Kemona A. PTP4A3 Expression Increases Strongly in Lymph Node Metastases from Colorectal Carcinoma. Anticancer Res. 2009;29:39133916.

[27] Vargas PA, Cheng Y, Barrett AW et al. Expression of Mcm-2, Ki-67 and geminin in benign and malignant salivary gland tumours. J Oral Pathol Med. 2008;37:309-18.

[28] Szajerka A, Dziegiel P, Szajerka T. et al. Immunohistochemical evaluation of metallothionein, Mcm-2 and Ki-67 antigen expression in tumors of the adrenal cortex. Anticancer Res. 2008;28:2959-65.

[29] Hashimoto K, Araki K, Osaki M. et al. MCM2 and Ki-67 expression in human lung adenocarcinoma: prognostic implications. Pathobiology. 2004;71:193-200.

[30] Yang J, Ramnath N, Moysich KB. et al. Prognostic significance of MCM2, Ki-67 and gelsolin in non-small cell lung cancer. BMC Cancer. 2006;6:203.

[31] Dudderidge TJ, Stoeber K, Loddo M. et al. Mcm2, Geminin, and KI67 define proliferative state and are prognostic markers in renal cell carcinoma. Clin Cancer Res. 2005;11:25102517.

[32] Kodani I, Shomori K, Osaki M. et al. Expression of minichromosome maintenance 2 (MCM2), Ki-67, and cell-cycle-related molecules, and apoptosis in the normal-dysplasia-carcinoma sequence of the oral mucosa. Pathobiology. 2001;69:150158.

[33] Czyzewska J, Guzinska-Ustymowicz K, Pryczynicz A, et al. Immunohistochemical evaluation of Ki-67, PCNA and MCM2 proteins proliferation index (PI) in advanced gastric cancer. Folia Histochem Cytobiol. 2009;47:289-296.

[34] Reena RM, Mastura M, Siti-Aishah MA. et al. Minichromosome maintenance protein 2 is a reliable proliferative marker in breast carcinoma. Ann Diagn Pathol. 2008;12:340-343.

[35] Gonzalez MA, Pinder SE, Callagy G. et al. Minichromosome maintenance protein 2 is a strong independent prognostic marker in breast cancer. J Clin Oncol. 2003;21(23):4306-4313.

Submitted: 4 February, 2010 Accepted after reviews: 11 August, 2010 